\title{
A (naïve) glance at Soft Computing *
}

\author{
Enric Trillas \\ Fundamentals for Soft Computing Unit \\ European Centre for Soft Computing \\ C/ Gonzalo Gutierrez Quirós , s/n \\ Mieres, Asturias 33600, Spain \\ enric.trillas@softcomputing.es \\ Claudio Moraga \\ Fundamentals for Soft Computing Unit \\ European Centre for Soft Computing \\ C/ Gonzalo Gutierrez Quirós , s/n \\ Mieres, Asturias 33600, Spain \\ Claudio.moraga@softcomputing.es \\ Sergio Guadarrama ${ }^{\dagger}$ \\ Fundamentals for Soft Computing Unit \\ European Centre for Soft Computing \\ C/ Gonzalo Gutierrez Quirós , s/n \\ Mieres, Asturias 33600, Spain \\ Sergio.guadarrama@softcomputing.es \\ Received: 05-11-2009 \\ Accepted: 28-05-2010
}

\begin{abstract}
This paper contains some naïve reflections on the currently large field of Soft Computing. After some general considerations, and of recalling some successful well known examples, it is posed the relationship between Soft Computing and Machine Intelligence. Finally, and with the aim of taking into account the appropriateness of Soft Computing to face the challenge of Computing with Words, some aspects of the semantical analysis of complex texts are considered.
\end{abstract}

Keywords: Soft Computing, Hybridization, Nature-inspired Methodologies, Machine Intelligence, Computing with Words.

\section{Preface}

In the opening paper ${ }^{1}$ to this Special Issue, "What is Soft Computing? Revisiting possible answers" Luis Magdalena expresses his views on this area of research. Motivated by his statements, the authors of the present contribution have tried to present their own experiences from the point of view of their Soft Computing related research work along the years.

Soft Computing mainly deals with imprecision, uncertainty, learning, and evolution, in posing and solving computational problems. Often, the solutions obtained for these problems are neither optimal, nor the most precise ones, nor the less uncertain, but suboptimal solutions. Good enough solutions that have

\footnotetext{
* This work has been supported by the Foundation for the Advancement of Soft Computing (ECSC) (Asturias, Spain).

${ }^{\dagger}$ Corresponding author
} 
'tractability, robustness, low solution cost, and better rapport with reality' in Zadeh's words ${ }^{2}$.

After the nineties of the last Century, the 'hybridization' of diverse methodologies became one of the main characteristics of Soft Computing. Hybridization started with neuro-fuzzy systems and continued with fuzzyevolutive ones to finally reach hybrid systems, integrating as many aspects of Soft Computing as needed, to be best adapted to the demands of the real problems at hand.

One of the future challenges for Soft Computing is to collide with 'Computing with Words', to make possible the integration of measurements and perceptions of reality that, once described in natural language, can be considered as data carrying intrinsically imprecise information. To properly afford this challenge, a lot of theoretic and empiric studies should be done to allow, for instance, the representation of linguistic expressions ${ }^{3}$ which are more complex than the semi-linguistic ones that are typical of Fuzzy Control ${ }^{4}$.

\section{General considerations}

In many industrial cases and internet-related services, technology does face problems of increasing complexity. Such complexity grows at a pace that is neither followed at the same rhythm by the performance power of theoretical and practical developments, nor by that of computational tools. As a consequence, new problems present challenging demands to those developments and tools that, at each moment, are at our hands.

One of the reasons for the difficulties to afford such technological problems with the existing 'classical' tools, is the fact that some systems can only be described, or this is more easily done, by means of semi-linguistic expressions involving imprecise, uncertain, and sometimes non-statistically describable concepts. Concepts that play, notwithstanding, a crucial role in the behaviour of the considered system. Classical tools are not well suited for dealing with such kind of linguistic expressions.

In these cases, Soft Computing offers the possibility of combining methods in a synergetic way to face the demands of the problem (see Ref. 5, 6, 7, 8). Different parts of the problem may be managed, or solved, by a different but complementary methodology or a combination thereof. For example, one part by classical methodologies, another one by either probabilistic, or possibilistic methodologies, another by using evolutionary algorithms, another by posing it in the framework of neural nets, and by using fuzzy logic, particularly for modelling what presents intrinsic imprecision.

Soft Computing methodologies offer solutions that, fitting with the practical granularity needed by the applications, are robust enough, and may reach lower costs than it could be expected if posed and solved with 'classical' pre-existent methodologies. Particularly in the area of fuzzy control ${ }^{9}$, and applications of neurofuzzy systems, a good number of solutions have reached industrial acceptance and realization ${ }^{10}$.

Soft Computing solutions can often be considered as not the best ones, from a scientific point of view, but they are usually reachable sub-optimal ones, that are good enough from a technological/engineering point of view. Moreover, let it be recalled that NP-complete problems are not solvable by classical methods within a reasonable computing time, meanwhile solutions have been obtained using the method called Ant Colony Optimization ${ }^{11}$.

There is, perhaps, a difficulty for the acceptance of tools coming from Soft Computing, which arises from the fact that 'classical' tools, as well as their theoretical grounds, usually were acquired along the education process at the Engineering Schools. Sometimes, this makes difficult for the working engineer to adapt her/his mind to new tools coming from a different way of posing and solving the problems that, in addition, lies on some non previously learnt theoretical basis. This is, in part, a reason for some reluctance to abandon the classical methodologies, and that can conduct to refuse those offered by Soft Computing, and even to pose the new kind of problems. In such reluctance also plays an important role the 'cultural belief' on the scientific necessity of managing only strictly precise variables.

\section{Recalling examples}

A first complex example that should be mentioned is the automatization of the control of the plants for sewage treatment, a problem for which there is a part with a not known mathematical model, but that is satisfactorily posed and solved by means of the fuzzy control methodology. Before such automatization, water purification was done by people with an expert knowledge that, once captured by a system of semilinguistic rules and translated into fuzzy terms, allowed 
the control of the plants in a technologically simple, robust and economical way ${ }^{12}$. Another even more complex example is the automatic control of a voicedriven helicopter ${ }^{13}$. A helicopter represents a physical system with a highly unstable behaviour that can fall down with just a few seconds delay in a pilot operation. For the automatization of the helicopter's movements not only were used the techniques of fuzzy control, but also of-line trained neural nets -(as highly effective, fast, non-linear signal classifier circuits)- because of the required short time of response needed to keep the flying helicopter under control. Possibly, the control by oral commands of a helicopter was the first complex case in which it did appear the central idea of Soft Computing in applications, namely, that of joining neural-nets and fuzzy control ${ }^{12}$.

The development of an interpretable model for a system from which only numerical data of behaviour is available, is a problem that can be very effectively solved with methods of Soft Computing by using e.g. a neural network to learn a model from the data, followed by a translation of the architecture of the neural network into a set of fuzzy if-then rules, which may finally be fine-tuned by means of an evolutionary algorithm. The synergy among a neural network, a fuzzy rule base and an evolutionary algorithm represents the very core of Soft Computing ${ }^{7}$. Evolutionary fine tuning of fuzzy rule based systems has already a long history ${ }^{14}$. Evolutionary fine tuning of the operations used by the rules of a fuzzy rule based system has been recently reported in Ref. 15. The leading idea of searching for synergy has approached Soft Computing to other disciplines. From the initial works with fuzzy logic, neural networks, genetic algorithms and evolutionary strategies, nowadays Soft Computing comprises a family of "bioinspired" or "nature-inspired" algorithms ${ }^{11}$. There are realizations of fuzzy controllers and fuzzy connectives in hardware as well as realizations of neural networks using e.g. FPGA technologies (see Refs. 17, 18, 19). Moreover, there has been an important opening towards Statistics when developing ensembles of neural networks ${ }^{20}$, and support vectors machines ${ }^{21,22}$.

\section{Soft Computing and Machine Intelligence}

In general what is easy for humans is quite difficult for machines, for example: No person is considered intelligent for being able to walk, run, talk, see, write, etc. These skills are too common to be considered in defining intelligence, which is in turn defined on not so common cognitive skills, such us, play chess, mathematics, spatial reasoning, novel writing, etc. Intelligence has been considered a scarce quality that only a few group of people have in high degree. On the other hand some things that computers do easily and fast are very difficult for humans, especially large computations and data storage.

The key role of Soft Computing is to help to define and develop systems with high Machine Intelligence. Although setting a proper definition could be impossible, some tentative measurements based on different sets of problems could serve for this purpose. Also what we expect and want from computers can serve to settle these measurements, since they can serve as a guide for researchers. For example, if we want computers able to communicate and interact with humans, then we should include these problems in the poll.

The interdisciplinary field of Soft Computing differs from conventional (hard) computing in that, unlike traditional computing, it is tolerant of imprecision, uncertainty, partial truth, and approximation. In many ways, Soft Computing represents a shift in the aims of computing, in that it simulates to some extent, the way the human mind works ${ }^{23}$. In particular, researchers in Soft Computing use to say that the inspirational models are the human mind, natural evolution and biological processes, which, unlike present day computers, do have a remarkable ability to process information which is pervasively imprecise, uncertain and lacking in rigid classes.

Soft computing is not a homogeneous body of concepts and techniques, but a guiding principle and a hybridization of methodologies ${ }^{24}$. Soft Computing refers to a collection of new computational techniques in computer science (and many applied and engineering areas) which study, analyze, and model very complex phenomena, those for which more precise scientific tools of the past were incapable of giving a solution.

\section{A partial look into the future}

Semantic aspects of texts represent an area where Soft Computing may make substantial contributions. For the narrative intelligibility of a text, its internal coherence is of great importance. In the process of writing a text, each piece of it should be coherent with the precedent ones and, in particular, they should exhibit a big degree 
of truth relative to the context created by the precedent ones, and considering also the restrictions imposed by the style of the writer. A truth value below some threshold could be a sign of non-intelligibility. In some sense, a process for checking the 'internal coherence' is similar to that of checking the correctness of a mathematical proof, in relationship with the syntactical rules of deduction followed in mathematics. In the case of a literary text, almost everything is of a semantic and context dependent character, and entails much more degrees of freedom for the writer.

Syntactical creative writing is nothing new. In the midfifties of the last Century, Newell and Simon's AI program 'Logical Theorist', did a proof of a theorem in the Russell and Whitehead's 'Principia Mathematica' shorter than the one in the book. Apart of the obvious differences between writing the proof of a theorem and other creative texts, what is actually in the future is what could be called 'semantic (complex)(creative) writing', like the writing of a novel by a computer. (In the meantime, the program SCIgen ${ }^{25}$ may be considered as an example of what is possible. This program generates "pseudo-scientific papers", which are used to put in evidence the bad quality of some "international conferences". The computer-generated papers do not really have a content, but they "sound" and "look" like real papers.) Another subject that concerns 'semantic writing' is the possibility of a computer system embodied in the writer's PC, helping the author to stepby-step check the successive pieces of text s/he is writing, for advising on the agreement or not agreement of such pieces with those written before; to advise her/him on the 'internal coherence' of the text by a computational semantic analyzer.

In looking ahead for such possible computational tasks, it should be taken into account that since usually creative writing involves linguistic expressions that are, at least, imprecise or uncertain, Soft Computing may be an interesting methodology, if not the only available, for purposes like that of constructing programs that can "understand" stories.

There are texts of novels that were semantically analyzed by means of the current armamentarium of fuzzy logic (see ref. 4), representing the text by means of its translation into fuzzy formulas, like it is done with the rules, or conditional statements, of imprecise ruled systems in Fuzzy Control. But for more complex, or sophisticated, linguistic expressions, the texts could be non-representable by present days armamentarium. It can happen, for example, in cases in which the use, or meaning, of the particle 'and' is either non commutative, or non associative, or it can be only captured after capturing the meaning of the full linguistic expression where it occurs (see ref. 3). At this point, it is important to notice the fact that fuzzy representations always need a careful design ${ }^{4}$, and that, in the way towards Computing with Words ${ }^{2}$, the current standard algebras of fuzzy sets ${ }^{26}$ should be reconsidered $^{27}$, and generalized ${ }^{28}$, to deal with parts of language that are more subtle than those currently considered. At his respect, the importance of Soft Computing for this kind of problems is reinforced by thinking that, for instance, to learn the literary style of a given writer or to do content based classification of large documents, the use of Neural Nets could be very effective ${ }^{22}$.

\section{Conclusions}

Since Zadeh introduced the idea of Soft Computing, the field has been growing without arrest. For instance the 'Berkeley Initiative on Soft Computing' -BISC-' (http://www-bisc.cs.berkeley.edu/) was established by the University of California at Berkeley in 1991 under the direction of Lotfi A. Zadeh, and as an Industrial Liaison Program. Furthermore in 2006, and also following an idea coming from Zadeh, the 'Foundation for the Advancement of Soft Computing' and its first born, the 'European Centre for Soft Computing, ECSC' (http://www.softcomputing.es), were respectively created and established in Mieres (Asturias, Spain). Between these fifteen years, Soft Computing passed from the kingdom of ideas to a scientific and technological reality, and today it ensembles a worldwide community of researchers and practitioners, with many specialized journals and conferences.

Soft Computing could represent a "Paradigm Shift", since it involves a re-definition of the traditional understanding of intelligence. Soft Computing includes many of the characteristics that in the conquest of certainty, total truth and precision have been traditionally avoided and devalued, for example: imprecision, uncertainty, partial truth, and approximate reasoning. 


\section{Acknowledgements}

This work has been supported by the Foundation for the Advancement of Soft Computing (ECSC) (Asturias, Spain) and by the Spanish Department of Science and Innovation (MICINN) under project TIN2008-06890C02-01 and under program Juan de la Cierva JCI-20083531 and the European Social Fund.

\section{References}

1. L. Magdalena, What is Soft Computing? Revisiting Possible Answers, Int. Jour. of Computational Intelligence Systems, (included in this issue).

2. L.A. Zadeh, Fuzzy Logic = Computing with Words, IEEE Trans. on Fuzzy Systems 4, (1996) 103-111.

3. S. Guadarrama, E. Renedo, E. Trillas and I. GarciaHonrado, An inquiry on And. Towards semantic-based models, ECSC Research Report FSC-2009-6, (2009).

4. E. Trillas and S. Guadarrama, Fuzzy representations need a careful design, International Journal of General Systems (in press).

5. R.A. Aliev and R.R. Aliev, Soft Computing and its Applications. (World Scientific, Singapore, 2001).

6. A. Tettamanzi and M. Tomassini, Soft Computing. Integrating Evolutionary, Neural, and Fuzzy Systems. (Springer, Berlin, 2001).

7. J.-S.R. Jang, C.T. Sun and E. Mizutani, Neuro-Fuzzy and Soft Computing, (Prentice Hall, 1997).

8. Ch. Borgelt, F. Klawoon, R. Kruse and D. Nauk, NeuroFuzzy Systeme, (Vieweg-Verlag, Wiesbaden, 2003).

9. D. Driankov, H. Hellendoorn and M. Reinfrank, An introduction to fuzzy control. (Springer, Berlin, 2000).

10. Y. Dote and S.J. Ovaska, Industrial Applications of Soft Computning: A review. Proc. of the IEEE 89 (9) (2001) 1243-1265.

11. M. Dorigo and T. Stützle, Ant colony optimization. (MIT Press, 2004).

12. H.T. Nguyen and N.R. Prasad (Eds.), Fuzzy Modeling and Control. Selected works of M. Sugeno. (CRC Press, 1999).

13. M. Sugeno, Avances de la ingeniería fuzzy en Japón. In: Applicaciones de la Lógica Borrosa, eds. E.Trillas and J. Gutiérrez, (CSIC Press, Madrid, 1992). (In Spanish)

14. O. Cordón, F. Gomide, F. Herrera, F. Hoffmann and L. Magdalena: Ten years of genetic fuzzy systems: current framework and new trends. Fuzzy Sets and Systems 141,(2004) 5-31.

15. C. Moraga, M. Sugeno and E. Trillas, Optimization of fuzzy if-then rule bases by evolutionary tuning of the operations. Proc. 39th IEEE Int. Symp. on Multiplevalued Logic. (IEEE-CS-Press, 2009).

16. E. Trillas, S. Cubillo and J.L. Castro, Conjunction and disjunction in [0,1], Fuzzy Sets and Systems, 72 (1995) 155-165.
17. A. Kandel and G. Langholz, Fuzzy hardware. Architectures and applications. (Kluwer, Boston, 1998).

18. K.S. Lindsey and T. Lindblad, Review of Hardware Neural Networks: a user perspective. http://www.particle.kth.se/ lindsey/elba2html/elba2html. html

19. A. Cabrera, S. Sánchez-Solano, P. Brox, A. Barriga and R. Sendhadji, Hardware/Software co-design of configureble fuzzy control systems. Applied Soft Computing, 4 (3) (2004) 271-285.

20. C. Moraga, Multi-nets: new trends in neural networks. Int. Journal of Systemics, Cybernetics and Informatics 3 (3), (2008) 11-18.

21. V. Vapnik, The Nature of Statistical Learning Theory. (Springer, 1995).

22. T. Joachims, Learning to classify Text using Support Vector Machines. (Kluwer/Springer, 2002)

23. L.A. Zadeh, Fuzzy Logic, Neural Networks, and Soft Computing, Communications of the ACM, 37 (3) (1994) 77-84.

24. L.A. Zadeh, Some reflections on Soft Computing, granular computing and their roles in the conception, design and utilization of information /intelligent systems. Soft Computing- A fusion of foundations, methodologies and applications, 2 (1) (1998) 23-25.

25. SCIgen - An automatic CS paper generator. http://pdos.csail.mit.edu/scigen

26. A. Pradera, E. Trillas, S. Guadarrama and E. Renedo, On Fuzzy Set Theories, in Fuzzy Logic, eds. Paul P. Wang, Da Ruan, Etienne E. Kerre (Springer, 2007) vol 215, pp. 15-47.

27. E. Trillas, On a Model for the Meaning of Predicates (A naïve approach to the Genesis of Fuzzy Sets), in Views on Fuzzy Sets and Systems from different perspectives, eds. R. Seising (Springer, 2009) vol. 243 pp. 175-205.

28. E. Trillas, C. Alsina, A. Pradera, On a Class of Fuzzy Set Theories, Proc. of FUZZ-IEEE 07, (2007) 1-5. 